\title{
Measuring fast ions in fusion plasmas with neutron diagnostics at JET
}

Eriksson, J.; Hellesen, C.; Binda, F.; Cecconello, M.; Conroy, S.; Ericsson, G.; Giacomelli, L.; Gorini, G.; Hjalmarsson, A.; Kiptily, V. G.

Total number of authors:

16

Published in:

Plasma Physics and Controlled Fusion

Link to article, DOI:

10.1088/1361-6587/aad8a6

Publication date:

2019

Document Version

Peer reviewed version

Link back to DTU Orbit

Citation (APA):

Eriksson, J., Hellesen, C., Binda, F., Cecconello, M., Conroy, S., Ericsson, G., Giacomelli, L., Gorini, G., Hjalmarsson, A., Kiptily, V. G., Mantsinen, M., Nocente, M., Sahlberg, A., Salewski, M., Sharapov, S., \& Tardocchi, M. (2019). Measuring fast ions in fusion plasmas with neutron diagnostics at JET. Plasma Physics and Controlled Fusion, 61, [014027]. https://doi.org/10.1088/1361-6587/aad8a6

\section{General rights}

Copyright and moral rights for the publications made accessible in the public portal are retained by the authors and/or other copyright owners and it is a condition of accessing publications that users recognise and abide by the legal requirements associated with these rights.

- Users may download and print one copy of any publication from the public portal for the purpose of private study or research.

- You may not further distribute the material or use it for any profit-making activity or commercial gain

- You may freely distribute the URL identifying the publication in the public portal 


\title{
Measuring fast ions in fusion plasmas with neutron diagnostics at JET
}

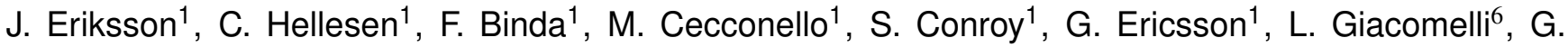
Gorini5,6, A. Hjalmarsson $^{1}$, V.G. Kiptily ${ }^{2}$, M. Mantsinen ${ }^{3,4}$, M. Nocente ${ }^{5,6}$, A. Sahlberg ${ }^{1}$, M. Salewski ${ }^{7}$, S. Sharapov², M Tardocchi' ${ }^{6}$ and JET Contributors*

${ }^{1}$ Department of physics and astronomy, Uppsala University, Sweden

${ }^{2}$ CCFE, Culham Science Centre, Abingdon, Oxfordshire, UK

${ }^{3}$ Barcelona Supercomputing Center (BSC), Barcelona, Spain

${ }^{4}$ ICREA, Pg. Lluís Companys 23, 08010 Barcelona, Spain

${ }^{5}$ Dipartimento di Fisica "G. Occhialini”, Universita' di Milano-Bicocca, Milano, Italy

${ }^{6}$ Istituto di Fisica del Plasma, Consiglio Nazionale delle Ricerche, Milano, Italy

${ }^{7}$ Department of Physics, Technical University of Denmark, Kgs. Lyngby, Denmark

\begin{abstract}
Fast ions in fusion plasmas often leave characteristic signatures in the neutron emission from the plasma. In this paper, we show how neutron measurements can be used to study fast ions and give examples of physics results obtained on present day tokamaks. The focus is on measurements with dedicated neutron spectrometers and with compact neutron detectors used in each channel of neutron profile monitors.

A measured neutron spectrum can be analyzed in several different ways, depending on the physics scenario under consideration. Gross features of a fast ion energy distribution can be studied by applying suitably chosen thresholds to the measured spectrum, thus probing ions with different energies. With this technique it is possible to study the interaction between fast ions and MHD activity, such as toroidal Alfvén eigenmodes and sawtooth instabilities. Quantitative comparisons with modeling can be performed by a direct computation of the neutron emission expected from a given fast ion distribution. Within this framework it is also possible to determine physics parameters, such as the supra-thermal fraction of the neutron emission, by fitting model parameters to the data. A detailed, model-independent estimate of the fast ion distribution can be obtained by analyzing the data in terms of velocity space weight functions. Using this method, fast ion distributions can be resolved in both energy and pitch by combining neutron and gamma-ray measurements obtained along several different sightlines.
\end{abstract}

Fast ion measurements of the type described in this paper will also be possible at ITER, provided that the spectrometers have the dynamic range required to resolve the fast ion spectral features in the presence of the dominating thermonuclear neutron emission. A dedicated high-resolution neutron spectrometer has been designed for this purpose.

\section{Introduction}

The most relevant reactions for the fusion research program are the $\mathrm{D}(\mathrm{d}, \mathrm{n})^{3} \mathrm{He}, \mathrm{D}(\mathrm{d}, \mathrm{p}) \mathrm{T}$ and $\mathrm{T}(\mathrm{d}, \mathrm{n})^{4} \mathrm{He}$ fusion reactions. These reactions are commonly referred to as the DD and DT reactions, respectively. Neutrons are produced in these reactions, which means that neutron measurements can be used to obtain information about the fuel ions in a fusion reactor. In this paper we consider neutron measurements from tokamak fusion experiments, which are sources of intense neutron emission;

*See the author list of "X. Litaudon et al 2017 Nucl. Fusion 57 102001" 
at the JET tokamak neutron rates of $5.5 \times 10^{16} \mathrm{~s}^{-1}$ and $5.7 \times 10^{18} \mathrm{~s}^{-1}$ have been achieved in D and DT plasmas, respectively.

The alpha particles $\left({ }^{4} \mathrm{He}\right)$ produced in the DT reaction carry 20 percent of the released fusion energy and will be an important source of plasma heating in a future electricity producing fusion reactor. In a tokamak, this "selfheating" is supplemented by externally applied heating methods, such as neutral beam injection (NBI) [1] and electromagnetic wave heating in, e.g., the ion cyclotron range of frequencies (ICRF) [2]. These heating methods rely on energy transfer to the bulk plasma during the slowing down of energetic ions with energies significantly higher than the average thermal energy. Such supra-thermal ions are commonly referred to as "fast ions", and their confinement in the plasma is of great importance in order to have effective plasma heating and high fusion performance. The physics of fast ions in fusion plasmas is therefore a topic of intense research [3-6].

This paper presents recent advances in the field of neutron based fast ion measurements. The aim is to give a coherent overview of this topic and connect results that have previously been presented separately. The paper is organized as follows. Section 2 contains a brief description of the measurement techniques relevant for this paper. In Section 3 we present the theoretical basis for neutron based fast ion measurements. Section 4 contains a presentation and discussion of selected physics results. In Section 5 a brief outlook about fast ion measurements in future high-performance fusion devices, such as ITER, is given. A summary and concluding remarks are presented in Section 6.

\section{Neutron measurement techniques}

The focus of this paper is measurements made with neutron spectrometers and neutron profile monitors (commonly called neutron cameras). In particular, most of the physics results discussed below are obtained at JET with the time-of-flight spectrometer TOFOR [7] and with the neutron camera [8]. Several other neutron spectrometers and cameras have been used on various tokamaks over the years. This includes dedicated spectrometer systems such as the magnetic proton recoil spectrometer at JET [9] and the time-of-flight spectrometer TOFED at EAST [10]; compact spectrometers based on diamond detectors [11,12] and scintillator detectors [13-15]; and neutron cameras at MAST [16], TFTR [17] and JT-60U [18].

Of key importance when analyzing data from any of the above instruments is the knowledge of the instrument response function, i.e. the expected measured signal due to a given neutron emission from the plasma. Depending on the instrument, the response function can be determined by particle transport modeling, measurements at a well characterized neutron source, or a combination of measurements and modeling. For a more detailed discussion about the response function for a specific instrument, the reader is referred to references [7-18] given above in connection with describing the respective instruments.

\section{Fast ion signatures in the neutron emis- sion from a fusion plasma}

In a given fusion reaction, the energy, emission direction and reaction probability of the emitted neutron depends on the momenta of the reacting fuel ions and on the angular differential cross section of the reaction [19]. Hence, the energy spectra and spatial emissivity profiles of DD and DT fusion neutrons are determined by the phase space distribution of the $\mathrm{D}$ and $\mathrm{T}$ ions. Fast ions often have distributions that are distinctly different from that of the thermal bulk plasma and therefore give rise to characteristic signatures in the neutron emission. These fast ion signatures can be used as the basis for diagnostics.

Fast ions gyrate around the plasma magnetic field lines with velocity $v_{\perp}$ and move freely along these lines with velocity $v_{\|}$. Consider a fast $\mathrm{D}$ population at a particular energy and pitch (i.e. $v_{\|} / v$ ) in a D plasma. The emitted neutron energy spectrum resulting from the fast ion population reacting with the thermal bulk plasma depends on (i) the fast ion energy, (ii) the fast ion pitch and (iii) the angle of observation relative to the plasma magnetic field. This is illustrated in Figure 1, where DD neutron energy spectra for different cases have been calculated according to the procedure described in [19]. The spectrum from thermonuclear reactions is well approximated by a comparatively narrow Gaussian [20], while the spectra from reactions between the fast ions and the bulk plasma typically cover a broader energy range and have a characteristic shape with two peaks. This feature 
is a result of the Doppler shift caused by the cyclotron gyration of the fast ions, and at a given ion energy it is most pronounced when the fast ions have $v_{\|} / v=0$ and are observed perpendicularly to the plasma magnetic field.

The spectrum calculations demonstrated above can be used to generate a complete map between the fast ion velocity coordinates and the neutron energy spectrum, e.g. by generating a grid of ion energies and pitch values and computing the neutron spectrum for each grid point. The result can be visualized in the form of velocity space weight functions, which show the region of the fast ion phase space that can give rise to neutrons in a given energy range. This practice is routinely used for FIDA [21, 22] and CTS measurements [23], and has recently been applied also to neutron diagnostics [24] and gamma-ray diagnostics [25,26]. Example weight functions for different neutron energy intervals are shown in Figure 2. Once the relevant weight functions have been computed, the neutron energy spectrum corresponding to reactions between an arbitrary fast ion distribution and the bulk plasma can be obtained by multiplying the weight functions with the fast ion distribution and integrating over the phase space coordinates. In practice this typically reduces to a number of matrix multiplications that can be rapidly evaluated. The instrument response function can also be taken into account in this process [27].

The neutron emission calculation techniques demonstrated above, in combination with the response function for the instrument under consideration, form the basis for the interpretation of all neutron measurements presented in this paper.

\section{Overview of recent results}

\subsection{Threshold analysis}

As a first basic example of how fast ion information is obtained from neutron measurements, we consider TOFOR spectrometer data from JET discharges 85372 and 85375. Data from two 1s time windows during these discharges are shown in Figure 3a. The TOFOR spectrum extends down to significantly lower times-of-flight for discharge 85372. Shorter time-of-flights correspond to higher neutron energies, and higher neutron energies are a sign of more energetic deuterons in the plasma, as seen from the weight functions in Figure 2. Based on the knowledge of the response function of TOFOR and from the kinematics of the fusion reactions between fast ions and the bulk plasma, it is thus possible to relate a given time-of-flight to a minimum deuteron energy [27]. This relationship is shown in Figure 3b. For instance, for JET discharge 85372 shown in Figure 3a the TOFOR data extends down to $\sim 50 \mathrm{~ns}$, which is direct evidence of the presence of deuterons with energies of at least $1 \mathrm{MeV}$ in the plasma. This result is attributed to ICRF acceleration of deuterons at the second harmonic of their cyclotron frequency [28]. For discharge 85375 on the other hand, no ICRF accelerated deuterons are observed; the data only extends down to about $58 \mathrm{~ns}$ (apart for some scattered points due to random background). Neutrons with this time-of-flight can be generated by deuterons with energies around $100 \mathrm{keV}$, i.e. the NBI energy at JET.

This kind of threshold analysis has been used to study resonant interactions between fast deuterons and toroidal Alfvén eigenmodes (TAEs), as reported in [29]. Here, TOFOR data from a JET discharge heated with a combination of deuterium NBI and ICRF tuned to the third harmonic of the deuterium cyclotron frequency was studied. With this heating scheme it was possible to accelerate deuterons up to several $\mathrm{MeV}$, which provided many opportunities to study fast ion physics. In particular, it was possible to study the interaction between the fast deuterons and TAEs by integrating the TOFOR data below various time-of-flight thresholds, thus probing fast deuterons above different deuteron energy levels. It was observed that the signal from fast deuterons with energies above $\sim 1 \mathrm{MeV}$ decreased significantly during periods of strong TAE activity, indicating that these ions were transported away from the plasma core (since this is the part of the plasma seen by TOFOR, c.f. Figure $3 c)$. Deuterons with energies below $\sim 0.5 \mathrm{MeV}$, on the other hand, were not affected by the TAEs. This energy dependent redistribution of the deuterons was found to be consistent with the expected location of different resonances between the deuterons and the TAE modes.

A similar analysis as above has recently been carried out with the JET neutron camera. In this case, the focus of the analysis was the behavior of fast deuterons during sawtooth instabilities. The plasma scenario studied was the same as in the example discussed above, i.e. third harmonic ICRF and NBI. By applying thresholds to the spectra measured by the different camera detec- 

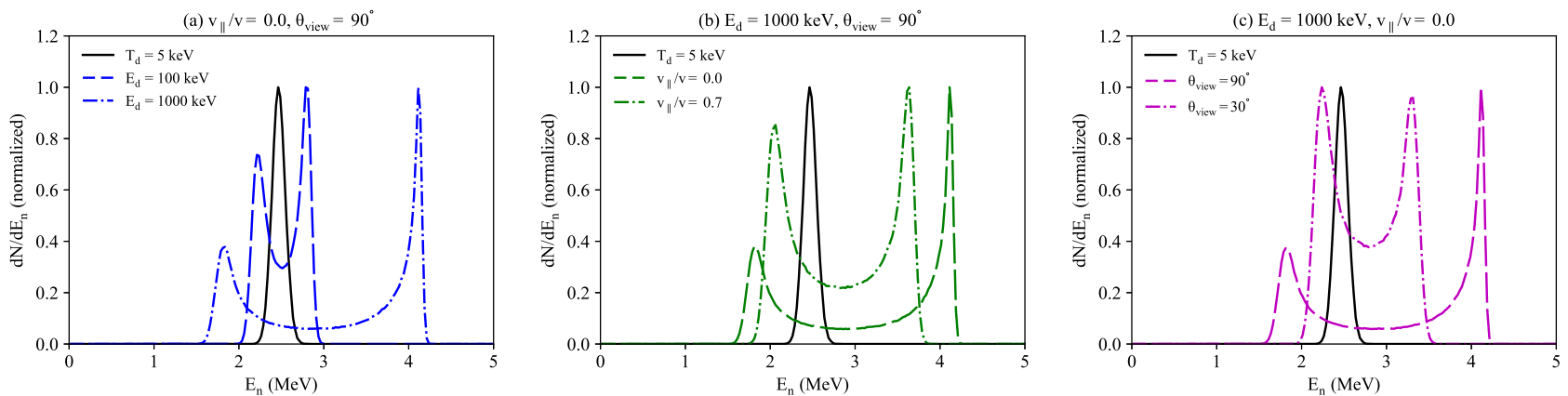

Figure 1: Calculated DD neutron energy spectra for reactions between different mono-energetic D distributions and a 5 $\mathrm{keV}$ bulk plasma (broken lines). Panel (a) demonstrates the effect of the fast $\mathrm{D}$ energy on the neutron spectrum, panel (b) demonstrates the effect of the fast $\mathrm{D}$ pitch and panel (c) demonstrates the effect of the viewing angle $\theta_{\text {view }}$ relative to the plasma magnetic field. The spectrum from purely thermo-nuclear reactions is also shown in all panels, for comparison (black solid line).
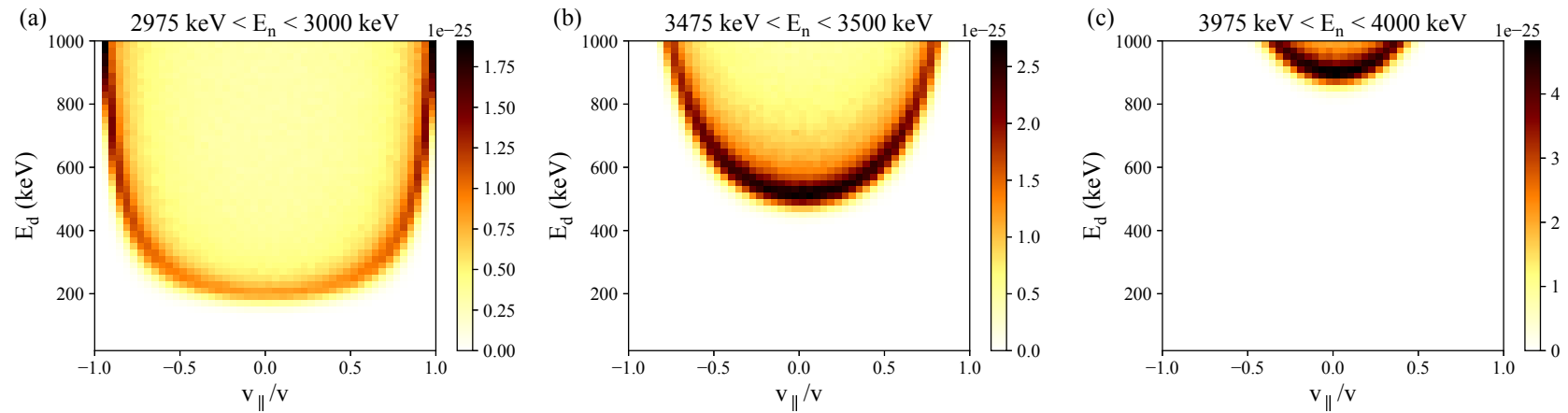

Figure 2: Example of DD weight functions for different neutron energy intervals, for reactions between fast deuterons and a $5 \mathrm{keV}$ bulk plasma. The viewing angle is perpendicular to the plasma magnetic field. Only fast deuterons in the colored regions of the plots can give rise to neutrons with energies in the given interval. (The color scale in these plots represent the number of neutrons emitted per solid angle per fast deuteron per unit bulk plasma density).

tors, it was possible to study the energy dependent redistribution of fast ions during sawtooth events. The observations are summarized in Figure 4, which shows data from the JET neutron camera before and after a sawtooth crash, using different thresholds for the energy deposited by the neutrons in the detectors. The lower threshold effectively probes deuterons of all energies, while the higher threshold only probes deuterons with energies above $\sim 1 \mathrm{MeV}$. It is observed that the relative difference between the pre and post sawtooth data is significantly smaller for the higher threshold setting, indicating that energetic deuterons (which are expected to be mainly trapped for this plasma scenario with strong ICRF acceleration) are less prone to be redistributed during a sawtooth event than ions with lower energies, in qualitative agreement with theory [30]. The effect is particularly clear in camera sightlines 4,5 and 15 , which are the ones viewing the most central regions of the plasma (c.g. Figure 4c). In these sightlines, a clear drop of the signal after the sawtooth is seen for the lower threshold
(Figure 4a), but no drop is seen for the high threshold (Figure 4b). A more detailed account of this study will be the topic of a future paper.

\subsection{Model validation and parameter fitting}

The analysis presented above relies only on basic considerations about fusion kinematics, along with knowledge of the response function of the neutron spectrometers under consideration. In order to perform more quantitative fast ion studies, one option is to use the methods described in Section 3 to calculate the neutron emission expected from a certain theoretical model or modeling code, and compare the calculations to the experimental data. Ideally, the calculations should be compared with as many different diagnostics as possible, in order to validate different aspects of the modeling. This is done e.g. in [31], where a fast D distribution computed with the ASCOT code [32] coupled to the RFOF library [33] is validated against several neutron and gamma-ray 

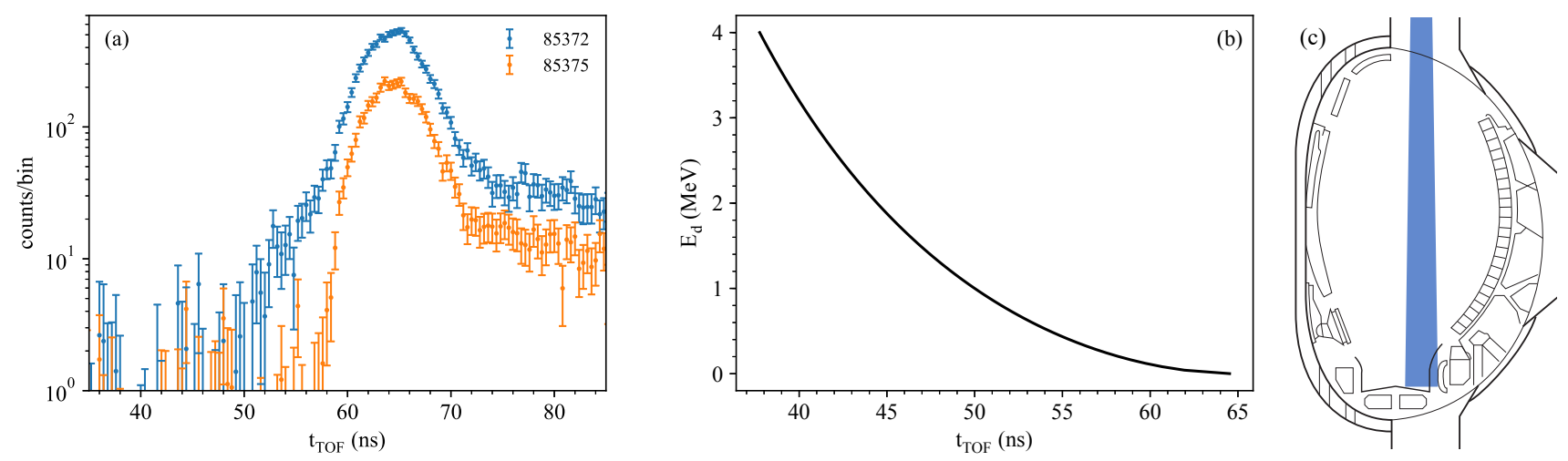

Figure 3: (a) Example TOFOR data from JET discharges 85372 (blue points) and 85375 (orange points). (b) Minimum deuteron energy required to give rise to a given time-of-flight in TOFOR. (c) Cross section of JET with the TOFOR field of view indicated (blue shaded region).
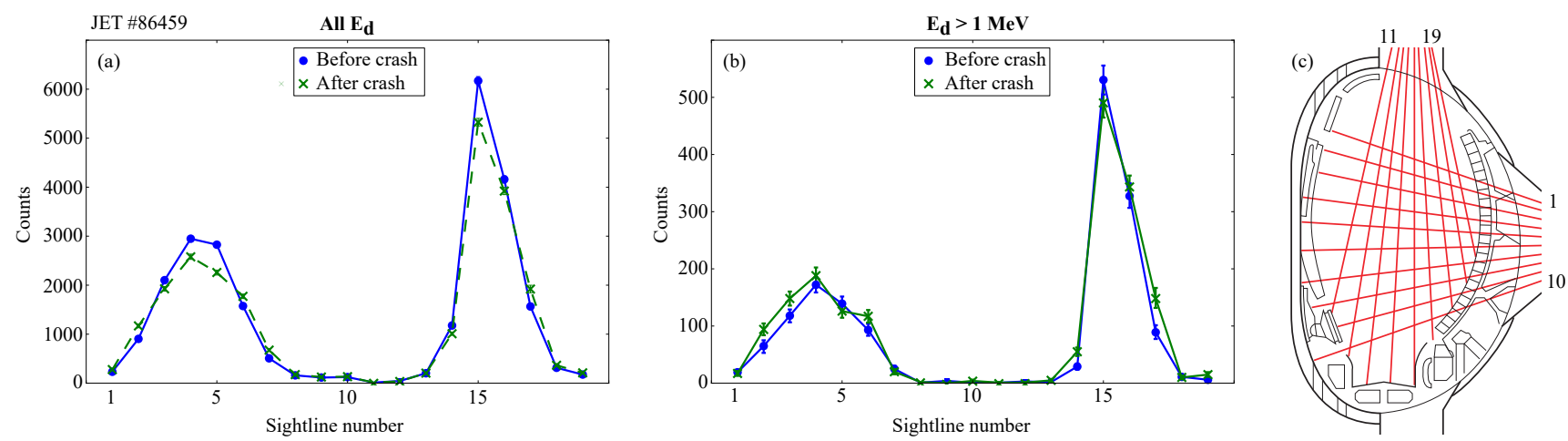

Figure 4: (a) Number of counts measured by each of the 19 JET neutron camera sightlines in a $90 \mathrm{~ms}$ time interval right before (blue dots) and after (green crosses) a sawtooth crash, for an energy deposition threshold of $2 \mathrm{MeV}$. With this threshold, deuterons of any energy can contribute to the neutron signal. (b) Same as in (a), but for an energy deposition threshold of $4 \mathrm{MeV}$. With this threshold, only deuterons with energies above $\sim 1 \mathrm{MeV}$ can contribute to the neutron signal. (Note that, for some data points, the error bars are smaller than the markers and thus not visible). (c) Cross section of JET with the camera sightlines indicated (red lines).

measurements. A summary of the results is presented in Figure 5, which shows the ASCOT fast D distribution and the comparison of the corresponding neutron spectra with data from the TOFOR spectrometer and an NE213 spectrometer. TOFOR has a vertical line-ofsight, viewing the plasma perpendicularly to the magnetic field, while the NE213 spectrometer has a horizontal line-of-sight that is oblique with respect to the magnetic field. As described in [31], this combination of diagnostics allows for validating the distribution obtained from the ASCOT-RFOF simulation in both energy and pitch, which would not have been possible with only one diagnostic. The plasma scenario under consideration is once again the 3rd harmonic ICRF scenario described above and the ASCOT-RFOF simulations indicate that this scenario results in deuterons accelerated to energies up to about $2 \mathrm{MeV}$ by the ICRF, with pitch values driven towards zero (c.f. Figure 5). The neutron spectra computed from this distribution agree well with the experimental data from both TOFOR and the NE213, which gives confidence in the modeled distribution.

A similar example of this kind of model validation has recently been carried out at MAST. In this case, a set of complementary fast ion diagnostics, including the MAST neutron camera, were used in conjunction with modeling to study fast ion behavior during TAEs and fishbone instabilities $[34,35]$. The MAST neutron camera has also been used in a similar way to study sawtooth redistribution [36].

Another possible analysis procedure is to start from a parameterized model of the deuterium distribution in the plasma and estimate the model parameters from a fit to 

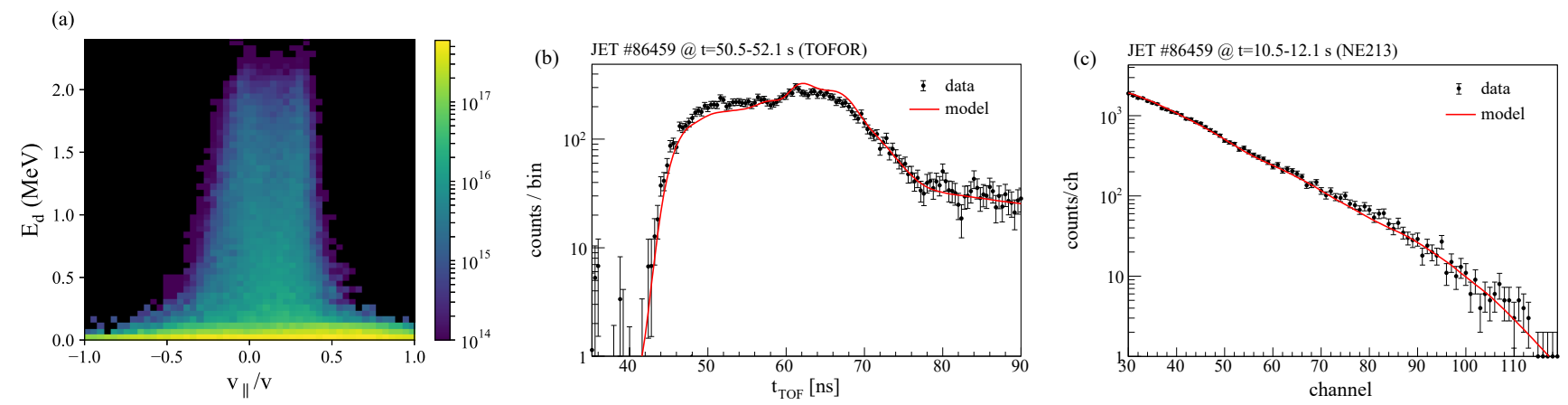

Figure 5: (a) Fast D distribution obtained from the ASCOT-RFOF code (color scale in arbitrary units). (b) The neutron spectrum computed based on this distribution compared to measurements with the TOFOR spectrometer (which has a vertical, perpendicular sightline). (c) The corresponding comparison with data from the NE213 spectrometer (which has a horizontal, oblique sightline). The comparisons in (b) and (c) is a validation only of the shape of the ASCOT distribution (the overall intensities of the calculated spectra has been fitted to match the data).

the experimental data. For example, the thermal bulk ion distribution is commonly modeled as a Maxwellian distribution, while distributions resulting from NBI and ICRF can be modeled e.g. with a 1-dimensional FokkerPlanck equation [2]. The overall normalization factors of the various distributions are often taken to be free parameters in the fit. When this kind of model is fitted to a measured neutron spectrum, it is thus possible to deduce the relative contribution that NBI ions and/or ICRF accelerated ions make to the neutron emission. This analysis technique has been extensively used in recent years e.g. to check for consistency between plasma heating codes and neutron measurements [37-39].

\subsection{Weight function analysis}

In the previous section it was described how neutron measurements can be used to validate modeled fast ion distributions, as well as to determine free parameters of a given model through a fitting procedure. A useful complement to these analysis methods is to use the velocity space weight functions described in Section 3 in order to obtain a model-independent estimate of the fast ion distribution. The basic idea of this method is to use the weight functions to generate "building blocks" for the measured spectra, where each building block represents a well localized region of the fast ion phase space (such building blocks will be referred to as " $\delta$-spectra" in what follows). The weights of all the $\delta$-spectra are then adjusted until the best match with the experimental data is found. The advantage of this modeling-independent approach is that it gives the possibility to resolve unknown or unexpected physics effects that might not be included in the available modeling codes. Also, if a modeled fast ion distribution is found to disagree with the data, a weight function based estimate of the distribution could give information about what part of the distribution that is causing the disagreement.

Any neutron measurement represents a volume integral over the viewing cone of the measuring instrument. This means that, in addition to velocity space, the weight functions should in principle be resolved also in real space. This can be achieved by computing weight functions in terms of a suitably chosen set of constants-ofmotion of the fast ions [40], and it is presently being investigated to what extent it is possible to apply this formalism to the full set of fast ion diagnostics available on JET.

However, in many situations, certain approximations about the weight functions can be justified, which reduces the complexity of the analysis. For instance, in many ICRF scenarios it is reasonable to assume that the fast ions are well localized to a comparatively small region in the core of the plasma, which makes it possible to consider the weight functions in terms of only the velocity coordinates of the fast ions. This approach was followed in [41], where the neutron and gamma-ray diagnostics at JET were combined in order to estimate the fast deuterium velocity distribution during the $3 \mathrm{rd}$ harmonic experiment described in Section 4.1. The resulting distribution was seen to be in good agreement with the distribution simulated with the ASCOT-RFOF code (c.f. Figure 5a), which is a further indication that ASCOT-RFOF reliably models the main features of the fast $\mathrm{D}$ distribution in this experiment. 
In addition to the assumption of a well localized fast ion distribution, it is sometimes also possible to make simplifying assumptions about the pitch values of the fast ions. In particular, for many ICRF scenarios, theory strongly suggests that the pitch of the accelerated ions are driven towards $v_{\|} / v=0$, since the ICRF wave field mainly accelerates the component of the ion velocity that is perpendicular to the magnetic field. Thus, assuming that the pitch values of the fast ions are narrowly distributed around zero effectively makes the weight functions depend only on the fast ion energy, as described in [42] (note that in this paper the term "weight functions" is never used explicitly).

As an example of such a one-dimensional weight function analysis we consider JET discharge 69247, which was heated with ICRF tuned to the 2nd harmonic of the D cyclotron frequency in combination with NBI heating. In this discharge, ICRF was applied continuously for about 10s. During this period, the NBI was switched on for two shorter time intervals of about $2 \mathrm{~s}$ each, with $3 \mathrm{~s}$ in between. Through a weight function based TOFOR analysis it was possible to estimate the time evolution of the fast $\mathrm{D}$ distribution, which allowed for a detailed comparison of the distribution during the combined ICRF+NBI and ICRF-only heating phases. The TOFOR distributions were also compared with modeled distributions obtained with the PION code [43]. A brief overview of the main results is given in Figure 6. Panel (a) shows the measured TOFOR data together with the spectrum estimated from the weight function analysis. A selection of the $\delta$-spectra used to build up the spectrum are also shown, for illustration. Each of the $\delta$-spectra corresponds to one deuteron energy and the deuteron energy distribution is thus obtained from the estimated weight of each $\delta$-spectrum. The resulting D energy distributions for periods with ICRF+NBI and ICRF-only is shown in panel (b) together with the corresponding distributions obtained from PION modeling. It is clear from these results that there are fewer energetic deuterons when the NBI is switched off, the biggest difference being in the region around $E_{\mathrm{d}}=100$ $\mathrm{keV}$. This result is expected, since the NBI ions provide a seed of moderately energetic particles that the ICRF can couple to, which results in the observed energetic tail of deuterons up to about $2 \mathrm{MeV}$. When the NBI is switched off, this seed disappears and the tail gradually decreases. These main features of the experimental ob- servations are also in good agreement with the PION modeling, although some discrepancy is seen, in particular in the high-energy tail of the NBI+ICRF distribution. A detailed discussion about the PION comparison is given in [44].

\section{Outlook towards ITER}

ITER will be equipped with a radial neutron camera (RNC) [45], allowing for measurements of the neutron emissivity profile (which is equivalent to the birth profile of the fusion alpha particles from the DT reaction). A conceptual design of a high-resolution neutron spectrometer (HRNS) is also available [46]. The HRNS is currently in the "enabled" category of ITER diagnostics, which means that it has an allocated sightline in the ITER design, but a definitive decision whether or not this instrument will actually be built at ITER is yet to be taken. The main plasma parameter to be measured by the HRNS is the fuel ion ratio $n_{\mathrm{T}} / n_{\mathrm{D}}$ in the core plasma, which is a critical parameter for machine protection and burn control. The HRNS is considered the primary diagnostic for performing this measurement. As discussed in [47], the determination of $n_{\mathrm{T}} / n_{\mathrm{D}}$ requires that thermonuclear and beam-target neutron emission components can be separated from each other in the measured neutron spectrum. I.e., even though the $n_{\mathrm{T}} / n_{\mathrm{D}}$ determination is not a fast ion measurement per se, it still requires that the $1 \mathrm{MeV}$ beam deuterons can be accurately measured.

In principle, all methods for measuring fast ions described in the previous section will be applicable also to ITER experiments. In addition to measuring D and T ions, it is also possible to measure alpha particles, which are manifested in the neutron spectrum due to elastic scattering on the fuel ions [48]. However, at ITER the plasma density and temperature is expected to be higher than in contemporary fusion experiments, which places higher demands on the dynamic range of an ITER neutron spectrometer compared to those of today. In a full power DT plasma at ITER it is likely that the intensity of the fast ion signatures in the neutron emission will be about 1000 times smaller than the thermonuclear intensity [47], which means that the dynamic range of the spectrometer needs to be at least three orders of magnitude. The scintillator detectors in the RNC sightlines will not meet this requirement unless the time integration is longer than $\sim 1 \mathrm{~s}$, due to limitations in count rate 
(a)

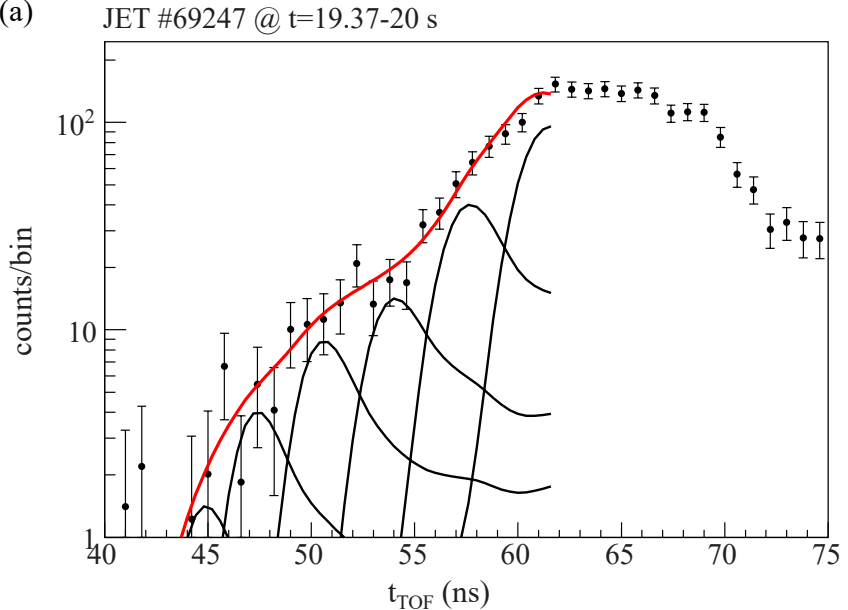

(b)

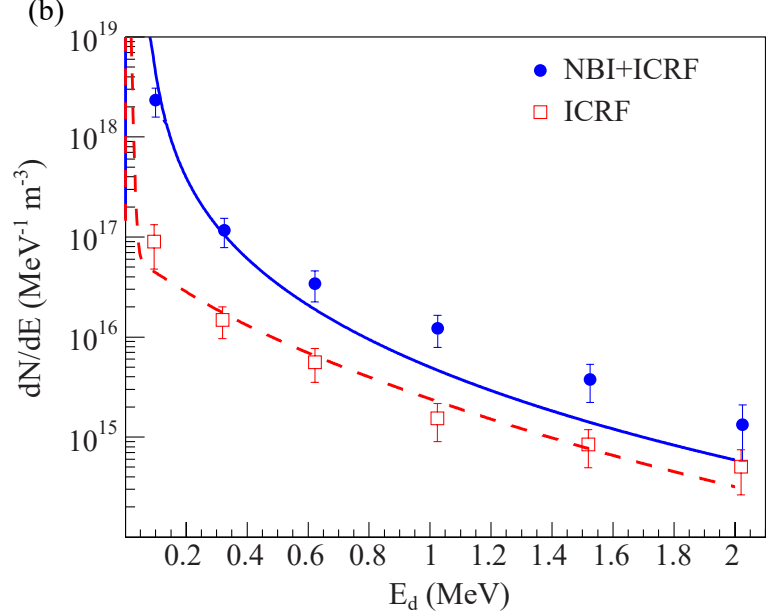

Figure 6: (a) TOFOR data (points with error bars) from a period of combined NBI and 2nd harmonic ICRF, together with the spectrum estimated from a 1-dimensional weight function analysis. A selection of the $\delta$-spectra used to build up the spectrum are shown as black lines and the total spectrum is shown in red. (b) D distributions for time windows with ICRF alone as well as in combination with NBI. The solid lines are the distributions obtained from PION simulations and the points represent the corresponding TOFOR results.

capability. However, with a dedicated spectrometer system, such as the HRNS, this dynamic range is attainable down to a time integration window of $100 \mathrm{~ms}$ [46], which conforms with the ITER requirements on the time resolution for $n_{\mathrm{T}} / n_{\mathrm{D}}$ measurements.

\section{Summary and conclusions}

Several of the most important fast ions in fusion plasmas (notably deuterons, tritons and, to some extent, alpha particles) give rise to neutrons emitted from the plasma. Neutron measurements can be used to study several different aspects of the physics of these fast ions. In particular, if a measurement resolves the energy spectrum of the emitted neutrons, it is possible to obtain detailed information about the distributions of the fast ions. Dedicated neutron spectrometers are installed on several major tokamaks, including JET, EAST and ASDEX-U. Furthermore, the compact scintillator detectors used e.g. in the neutron profile monitor at JET also have spectroscopic capabilities, which can now be exploited.

A measured neutron spectrum can be analyzed in several different ways, depending on the physics scenario under consideration. It is possible to set thresholds in the measured spectrum, in order to selectively probe fast ions in different energy regions. This approach has proved useful for studying transient phenomena that require high time resolution, such as the redistribution of fast ions during magnetohydrodynamic instabilities. Another op- tion is to perform direct comparisons between neutron measurements and plasma modeling by calculating the neutron emission expected from a given modeled fast ion distribution, thus aiding the physics interpretation of a given experiment. If the model contains free parameters, such as the normalization factors of the different ion distributions, it is possible to estimate these parameters by fitting them to the experimental data. By utilizing the concept of kinematic weight functions, which map the fast ion phase space to the possible neutron energies that the fast ions can give rise to, it is possible to make a model independent estimate of the fast ion distribution. This method allows for combining different fast ion diagnostics in a consistent way, by including the relevant weight functions for the respective diagnostics.

Fast ion measurements of the type described in this paper will be possible to perform with neutron spectroscopy also at ITER, provided that the spectrometers have a dynamic range of at least 3 orders of magnitude, in order to resolve the fast ion spectral features in the presence of the dominating thermonuclear neutron emission.

\section{Acknowledgments}

This work has been carried out within the framework of the EUROfusion Consortium and has received funding from the Euratom research and training programme 2014-2018 under grant agreement No 633053. The 
views and opinions expressed herein do not necessarily reflect those of the European Commission.

\section{References}

[1] Stix T H 1972 Plasma Physics 14367

[2] Stix T H 1975 Nuclear Fusion 15737

[3] ITER Physics Expert Group on Energetic Particles, Heating and Current Drive and ITER Physics Basis Editors $1999 \mathrm{Nu}$ clear Fusion 392471

[4] Heidbrink W W and Sadler G J 1994 Nuclear Fusion 34535

[5] Sharapov S E et al 2013 Nuclear Fusion 53104022

[6] McClements K G and Fredrickson E D 2017 Plasma Physics and Controlled Fusion $\mathbf{5 9} 053001$

[7] Gatu Johnson M et al 2008 Nuclear Instruments and Methods in Physics Research Section A: Accelerators, Spectrometers, Detectors and Associated Equipment 591 417-430

[8] Adams J et al 1993 Nuclear Instruments and Methods in Physics Research Section A: Accelerators, Spectrometers, Detectors and Associated Equipment 329 277-290

[9] Ericsson G et al 2001 Review of Scientific Instruments 72759

[10] Zhang X et al 2014 Nuclear Fusion 54104008

[11] Cazzaniga C et al 2014 Review of Scientific Instruments 85 043506

[12] Muraro A et al 2016 Review of Scientific Instruments 87 $11 \mathrm{D} 833$

[13] Binda F et al 2014 Review of Scientific Instruments 85 11E123

[14] Belli F et al 2012 IEEE Transactions on Nuclear Science 59 2512-2519

[15] Giacomelli L et al 2011 Review of Scientific Instruments 82 123504

[16] Cecconello M et al 2014 Nuclear Instruments and Methods in Physics Research Section A: Accelerators, Spectrometers, Detectors and Associated Equipment 75372 - 83

[17] von Goeler S et al 1996 Review of Scientific Instruments 67 473

[18] Ishikawa M et al 2002 Review of Scientific Instruments 73 $4237-4242$

[19] Eriksson J et al 2016 Computer Physics Communications 199 $40-46$

[20] Brysk H 1973 Plasma Physics 15611

[21] Heidbrink W W et al 2007 Plasma Physics and Controlled Fusion 491457
[22] Salewski M et al 2014 Plasma Physics and Controlled Fusion 56105005

[23] Salewski M et al 2011 Nuclear Fusion 51083014

[24] Jacobsen A et al 2015 Nuclear Fusion 55053013

[25] Salewski M et al 2015 Nuclear Fusion 55093029

[26] Salewski M et al 2016 Nuclear Fusion 56046009

[27] Jacobsen A S et al 2017 Review of Scientific Instruments 88 073506

[28] Eriksson J et al 2014 Europhysics Conference Abstracts 38F P1.023

[29] Hellesen C et al 2010 Nuclear Fusion 50084006

[30] Kolesnichenko Y I and Yakovenko Y V 1996 Nuclear Fusion 36 159-172

[31] Eriksson J et al 2015 Nuclear Fusion 55123026

[32] Hirvijoki E et al 2014 Computer Physics Communications 185 $1310-1321$

[33] Johnson T et al 2011 AIP Conference Proceedings 1406373 376

[34] Cecconello M et al 2015 Plasma Physics and Controlled Fusion 57014006

[35] Jones O M et al 2015 Plasma Physics and Controlled Fusion 57125009

[36] Cecconello M, Sperduti A and thê̂A MASTÂ team 2018 Plasma Physics and Controlled Fusion 60055008

[37] Hellesen C et al 2010 Plasma Physics and Controlled Fusion 52085013

[38] Mantsinen M J et al 2015 Europhysics Conference Abstracts 39E P2.171

[39] Gallart D et al 2017 EPJ Web of Conferences 15703015

[40] Stagner L and Heidbrink W W 2017 Physics of Plasmas 24 092505

[41] Salewski M et al 2017 Nuclear Fusion 57056001

[42] Hellesen C et al 2013 Nuclear Fusion 53113009

[43] Eriksson L G, Hellsten T and Willen U 1993 Nuclear Fusion 331037

[44] Hellesen C et al 2018 Nuclear Fusion 58056021

[45] Marocco D, Esposito B and Moro F 2012 Journal of Instrumentation $7 \mathrm{C} 03033$

[46] Scholz M et al 2018 'System Design Description Document (DDD) - High Resolution Neutron Spectrometer DDD-PBS 55.BB (Enabled)' Tech. rep. Fusion for Energy

[47] Hellesen C et al 2015 Nuclear Fusion 55023005

[48] Källne J et al 2000 Physical Review Letters 85 1246-1249 